\title{
Cognitive Behavior Therapy Improved the Symptoms of Bulimia Nervosa in the Athletes
}

\author{
${ }^{1}$ Evrim Çakmakçı, ${ }^{2}$ Majid Mozafari Zadeh, ${ }^{3}$ Seyed Morteza Tayebi* \\ ${ }^{1}$ Department of Coaching Education, Faculty of Sport Science, Selcuk University, Konya, Turkey. \\ ${ }^{2}$ Department of Sport Psychology, Allameh Tabataba'i University, Tehran, Iran. ${ }^{3}$ Department of Exercise \\ Physiology, Allameh Tabataba'i University, Tehran, Iran.
}

Submitted 19 August 2019; Accepted in final form 15 October 2019.

\begin{abstract}
Background. Different studies indicate a high prevalence of eating disorders in athletes. These disorders represent a severe defect in many areas of life. Eating disorders can lead to changes in consumer or intake of food and significant damage to physical health and psychosocial sports performance. Objectives. The aim of the present study was examining the effects of Cognitive Behavior Therapy (CBT) on the symptoms of Bulimia Nervosa (BN) of the Athletes. Methods. The research method was semi-experimental with pre-test, post-test, and three-month follow-up test. Three hundred athletes of endurance, aesthetics, and weight-based pre-match sports assessed using the Eating Disorder Diagnostic Scale (EDDS) via Structured Clinical Interview (SCID) and found sixteen athletes with BN. Then they assigned randomly to control and experimental groups. Experimental groups received CBT treatment based on Fairburn et al. (2008) protocol (4 steps, 21 sessions during 16 weeks). Twelve weeks after the final session of CBT, all two groups assessed again by EDDS via SCID to follow up on the persistence of CBT. Results. The symptoms of $B N$ is decreased significantly during CBT, but it didn't change in the control group $\left(\eta^{2}=0.69, \mathrm{f}_{2,28}=30.77, \mathrm{p}<0.001\right)$. On the other hand, the weight of athletes with BN symptoms increased significantly during CBT, but it decreased in the control group $\left(\eta^{2}=0.5, \mathrm{f}_{1.27,17.78}=14.27, \mathrm{p}=0.001\right)$. Conclusion. The results of the present study confirm the effectiveness of $\mathrm{CBT}$ on the improvement of BN symptoms and weight management, as well as its persistence in athletes.
\end{abstract}

\section{KEYWORDS: Cognitive Behavior Therapy, Eating Disorders, Bulimia Nervosa, Sports, Athletes, Weight Management}

\section{INTRODUCTION}

On the one hand, appropriate nutrition is a vital concept emphasized by parents, teachers, educators, and governments, because diet affects any organ in the body, and hence can significantly affect one's health. On the other hand, the community presents an image of body ideals that can be combined with genetic defects to cause eating disorders (1).

Eating disorders are debilitating syndromes characterized by severe disturbances in eating behaviors and extreme concerns about the body shape and weight that indicate deficits in many fields of life $(2,3)$. According to Diagnostic and Statistical Manual of Mental Illness (DSM), these disorders are characterized by persistent eating disorders or eating behaviors that lead to changes in food consumption or intake and significant damage to physical and mental health and social functioning (4); and include: pike, rumination disorder, avoidant food intake disorder, Anorexia Nervosa, Bulimia Nervosa, and Binge-eating Disorder according to Diagnostic and Statistical

*. Corresponding Author:

Seyed Morteza Tayebi, Assistant Professor

E-mail: tayebism@gmail.com 
Manual of Mental Illness (DSM) version V (4). Among these, Bulimia Nervosa is one of the most common types of eating disorders that have been reported in both clinical and non-clinical populations (5). It affects the youth and causes severe damage to quality of life and death in even more severe cases (6). In this regard, one of the communities in which eating disorders have attracted views is the sports community. Although some studies show that eating disorders are more common among athletes than the general population, the prevalence of these problems among athletes is approximately equal to the prevalence among the general public. As shown in several studies (7-14), in a competitive sport setting, there is a risk that the athlete may be excluded from the group program because of eating disorders. Therefore, athletes with this type of disease are usually very confidential, and until the problem turns into a catastrophe and there is a need for expert help, they are reluctant to share information. Anesthesia and dysuria, therefore, occur in exercise, so it is essential to understand the different patterns of an eating disorder that fall within this range (15). Several studies have been conducted in this regard. Kerr, Berman, and DeSouza found that among gymnasts still in work, the rate of reporting eating disorders was $3 \%$ and eating irregularity was $18 \%$, and 20 and $73 \%$ among retired gymnasts, respectively (16). Earlier, Kirchner, Lewis, and O'Connor concluded that college gymnasts had no high score on eating disorders and were concerned about their weight (17).

Bulimia Nervosa includes repeated binge eating, lack of control over eating behavior during binge eating period, regular, intentional vomiting, taking laxatives, strict diet, or vigorous exercise to prevent weight gain, on average two meals a week or period of at least three months, constant and overly concerned about body weight and shape. Those with Bulimia Nervosa usually develop depression due to low self-esteem, eating for an extreme sense of self, then conscience overeating, and deliberately vomit or take laxatives to remove food from their bodies $(5,18$, 19). In this regard, cognitive-behavioral therapy is recognized as the most effective treatment for Bulimia Nervosa and binge eating disorder. It also seems to be generalizable in the community of affected athletes.

The cognitive-behavioral therapy is a psychological intervention that is the most common evidence-based approach to improving mental health $(20,21)$. The Cognitive Behavioral Therapy Model for the Treatment of Eating Disorders is based on the principle that inefficient beliefs about body weight and dissatisfaction with body weight and shape lead to the development and continuation of extravagant eating behaviors and other characteristics such as vomiting and taking a laxative (21). The Cognitive Behavioral Therapy approach is necessarily multifactorial, and in addition to psychological training, selfreview, and cognitive and behavioral interventions, it also includes nutritional counseling (22). Turner (2016) pointed out that some athletes need to work at a deeper level to neutralize central irrational beliefs that lead to unhealthy behaviors and emotions and may thus be effectively treated through rational, emotional behavior therapy. Rational Emotive Behavioral Therapy is humanistic, so it focuses not only on the athlete and his athletic performance but also on the person; therefore, cognitive and emotional behavioral therapy addresses a wide range of muscular problems apart from functional issues such as career change, personal life problems and eating disorders (23).

Since eating disorders usually begin in adolescence (24) and the sufferer suffers greatly because these are associated with significant deficits in social adaptation and quality of life, including interpersonal relationships and job productivity. Besides, traumatic eating habits such as overeating, purging or restricting eating can also have negative consequences such as obesity, malnutrition and even demise, so these disorders are serious mental health problems and strive to understand better, prevent and treat these disorders seems necessary to reduce the individual and social harm (1). On the one hand, we know many athletes with eating disorders in different sports settings. Eating disorders and harmful weight control behaviors appear to be present not only in aesthetic and weight-based exercises but also in a wide range of sports. However, eating disorders are hidden in many athletes for various reasons. Probably one of the most important motivations for this is the feeling of shame. The athletes, coaches, parents, and all those who are some sportsman, even the spectators, hate things such as overeating and purging. This feeling of shame and disgust is further internalized among athletes who control weight and nutrition, helping them to avoid 
adverse emotional reactions from those who know their problem. Therefore, it is essential to pay attention to this issue in athletes $(25,26)$, and as stated, cognitive-behavioral therapy seems to be the most effective treatment for Bulimia Nervosa. So, the purpose of the present study is to investigate the effect of a cognitive-behavioral therapy course on the signs of Bulimia Nervosa in athletes.

\section{MATERIALS AND METHODS}

This study is a quasi-experimental study with pre-test, post-test and three-month follow-up test with the control group.

Participants. 16 athletes were selected from 300 athletes available in the fields of endurance (swimming and running), aesthetics (gymnastics), weight-based pre-match (taekwondo and wrestling) and club (bodybuilding) sports in Tehran aged 16 to 28 years who received the highest score from a structured clinical interview for accurate diagnosis of patients with Bulimia Nervosa disorders for this study. Two experienced psychologists confirmed the subjects with eating disorders. These 16 boys and girls athletes (11 girls and five boys) with a mean age of 20 years and a mean of 4 years of presence at semiprofessional and professional levels were randomly divided into two groups. Eight athletes (five girls, three boys) were selected for the experimental group, and eight athletes (six girls and two boys) were selected for the control group. Two of the best athletes were selected, one for the experimental group and the other for the control group. The next two top athletes were placed in the same order and were then placed in the same experimental and control groups. All subjects were assured that all their information would be confidential and that the information obtained would be used solely for research.

Tools and Data collection. Bulimia Nervosa was evaluated through the Eating Disorder Diagnostic Scale (EDDS). The data were collected using a semi-structured clinical interview for Semi-Structured Clinical Interview for DSM-IV Axis I Disorders (SCID-I).

Semi-structured Interview for DSM-IV Axis I Disorders (SCID-I). An interview is a flexible tool developed by First et al. (27). Amini et al. for the validation of the Farsi version of SCID-I, reported that it is a valid tool for diagnosis for clinical purposes, especially for research and even training; therefore, it is recommended to use it for the above purposes in clinical settings (28).

Eating Disorders Detection Scale (EDDS). Eating Disorders Detection Scale (EDDS) for Eating Disorders is a 22-item questionnaire that measures Anorexia Nervosa, Bulimia Nervosa, and binge eating disorder based on criteria of Diagnostic and Statistical Manual of Mental Disorders. This scale consists of a combination of Likert scores, 2-part scores, Frequency scores, and open-ended questions such as height and weight (29).

First, the ethical and technical issues of the research were reviewed and approved by two experienced psychologists. To reach the athletes. First we contacted the coaches and officials of the clubs, and the athletes were evaluated using a convenience sampling method. After the initial examination and selection of 16 eligible subjects, the appropriate time and place for the initial appointment were coordinated. At the initial meeting, the initial talks were about starting an interview and treatment. The athletes under 18 years old provided parental consent. The athletes were informed about the stages and nature of this study. The athletes expressed readiness to complete the research and completed the informed consent form. The exact dates of all sessions (pre-test, post-test, follow-up, and training sessions) were determined and scheduled. The questionnaires were completed once before the start of the Cognitive Behavioral Therapy sessions (pre-test), once immediately after the sessions (post-test), and once 12 weeks after the completion of the Cognitive-Behavioral Therapy (Follow-up) sessions. Since psychological validity, reliability and accuracy of the interviews are very important, so the reliability and validity of the first interview were re-confirmed. The subject was screened using a mental health screening for 7 minutes (Mohammadi et al., 2016), followed by details on SCID, which is a structured interview, and it was shown that the subject was suffering from Bulimia Nervosa. Research data were collected using the Eating Disorders Detection Scale (EDDS).

Treatment Sessions. The present study used the therapeutic protocol of Fairburn (2008) (30). It is crucial that from the beginning of treatment, the patient is in a position to receive optimal treatment. For this reason, any potential barriers to the development of advanced cognitive- 
behavioral therapy should be considered. The significant contraindications for treatment initiation are physical features of concern, severe clinical depression, considerable drug abuse, distracting life events, and lack of commitment. Such factors should be considered before starting treatment.

Stage 1. Treatment must begin well. This results is consistent with evidence that the rate of changes early in treatment is well predictive of treatment results. This intensive early stage is designed to achieve first movement rate of treatment, comprising approximately eight sessions twice a week for four weeks. The purpose of Stage 1 is to build patient engagement in treatment and change to provide a personalized formulation for treatment and disorder training, and introduce and implement two essential approaches: "weekly weight" engagement and regular eating. The changes at this stage of treatment form the basis for other changes.

Stage 2. This stage is brief but essential, the transitional or transition stage generally consisting of two appointments (2 sessions, one session each week). While continuing with the methods described at Stage 1, at Stage 2 the therapist and patient think carefully about what has happened and decide for the next move and follow up on progress, aimed to identify problems and obstacles that emerge in the way of change. In order to enter the third stage, in the case of formulation if necessary, appeal is done for a number of purposes. If patients are making good progress, it is better to acknowledge their efforts and to encourage useful change. If patients do not do well, explanations need to be understood and re-introduced. If clinical perfectionism, low selfesteem, and communication problems appear, this is a sign of a broader range of treatments.

Stage 3. This step is the main stage of treatment. Its purpose is to introduce the essential processes that support the patient's eating disorder. The mechanisms have been introduced, and the order in which they are encountered depends on their relative role and importance in maintaining the patient's psychopathology. This stage is generally eight weekly sessions, with one meeting per week ( 8 weeks).

Introducing over-evaluation of shape and weight and the results

The first step involves describing the concept of self-assessment and helping patients identify how they evaluate themselves. The relative importance of the various relevant fields may be shown as a baseline diagram. The patient and therapist then identify problems with this selfassessment program. In summary, there are three related problems: first, over-evaluation depends on performance in a field of life, with domains other than marginal shape and weight; second, shape and weight control where success is beyond reach so undermining self-esteem; and third, over-evaluation is responsible for the behavior that describes eating disorders (dieting, binge eating, etc.). The last step is to consider selfassessment for the advanced formulation, defining the basic terms of overweight and body evaluation: dieting, body examination, and physical avoidance, obesity, and marginalizing other fields of life. The therapist uses advanced formulation to describe how these behaviors and experiences are used to maintain and magnify the patient's concerns about shape and weight, and thus they are needed for treatment introduction.

Stage 4. The last step is treatment and is termed as the end of proper treatment. The focus is on keeping track of the progress that has been made and preventing and reducing mistakes that also called mistake prevention. Usually three appointments take place in two weeks. During this stage, since patients prepare for the end of treatment, patients do not continue their review and begin weightlifting at home. To maximize the chance of maintaining progress, the therapist and the patient jointly plan a personal plan for the months ahead until the post-test. Typically it involves working more on physical examination, avoiding eating, and maybe problem-solving exercises. Also, patients are encouraged to continue their efforts to develop new interests and activities. There are two elements for reducing the risk of mistakes. First, patients need to have realistic expectations about the future. Not expecting any painful experiences again in eating disorders makes the patient vulnerable to the mistake because it encourages the patient to react negatively to minor problems. Instead, patients should consider eating problems as their Achilles tendon.

The purpose is for patients to identify their small issues as early as possible, view them as problems rather than mistakes, and actively identify them as strategies learned during treatment.

Statistical Analysis. Statistical analysis was performed using repeated-measures ANOVA $(2 *$ 
3). All results were presented with error level $\alpha<$ 0.05 . Software SPSS was used for data analysis.

\section{RESULTS}

Among eight participants, five participants $(62.5 \%)$ were female, and three participants $(37.5 \%)$ were male, and in the control group, six participants $(75 \%)$ were female, and two participants $(25 \%)$ were male.

Weight. The composite symmetry assumption for the variable of weight was not met ( $p=0.004$, $\mathrm{w}=0.426$ ); hence, considering GreenhouseGiesser correction the group interaction and time effect were significant (effect size $=0.5, \mathrm{p}=$ $0.001,14.27, \mathrm{~F}=17.78,1.27$ and 14.27 , so that the effect was linear (effect size $=0.46, p=0.004$, $\mathrm{F}=1.78,11.1$, and 14) and square (effect size $=$ $0.58, \mathrm{p}=0.001, \mathrm{~F}=27,19.1$, and 14) (Fig. 1); in other words, when athletes with Bulimia Nervosa under cognitive-behavioral therapy experienced weight gain during the treatment period, the athletes in the control group had the weight loss disorder. On the other hand, at the end of the treatment period and during the follow-up period, the two groups as the post-test remained unchanged.

The signs of Bulimia Nervosa. The interactive effect of group and time was significant, with composite symmetry assumption $(\mathrm{p}=0.6, \mathrm{w}=0.938)($ effect size $=0.69, \mathrm{p}<0.001$, $F_{2,28}=30.77$ ), so that the effect was linear (effect size $\left.=0.8, \mathrm{p}>0.001, \mathrm{~F}_{1,14}=58.03\right)$ and square (effect size $\left.=0.5, \mathrm{p}=0.002, \mathrm{~F}_{1,14}=13.96\right) .13$ ) (Fig. 2); in other words, when athletes with Bulimia Nervosa under cognitive behavioral therapy experienced weight loss during the treatment period, the control group athletes showed no change. On the other hand, after the end of the treatment period and during the followup period, the signs of Bulimia Nervosa in the two groups as the post-test remained unchanged.

\section{DISCUSSION}

This study was conducted aimed to examine the effectiveness of cognitive-behavioral therapy on the signs of athlete's Bulimia Nervosa. To the best of our knowledge, no report has been observed that directly treats Bulimia Nervosa in athletes through cognitive-behavioral therapy, but in the non-athlete community, few studies investigated the effect of cognitive factors on eating disorders. So the present study is the first official study in this field in the athletic community. Other studies related to the subject of the present study, which has been conducted in the past, have focused on the prevalence of eating disorders, with the results suggesting a high incidence of eating disorders among athletes; considered psychological problems as the most critical problems of athletes $(7,31-33)$ such as Rosen and Hough (1986) Brewer and Petrie (2002), Pernick et al. (2006), and Petrie et al. (2008).

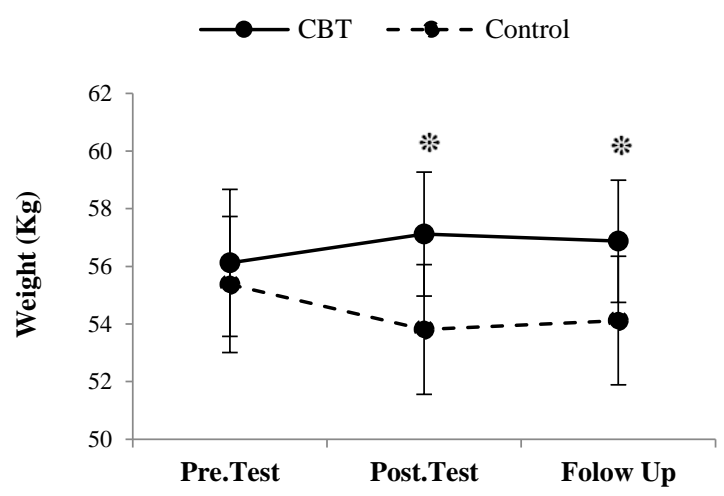

Figure 1. The Effect of Cognitive-behavioral Therapy on the Weight of Athletes with Bulimia Nervosa. *: Significant Interactive Impact of Group and Time at $p<0.001$ Level
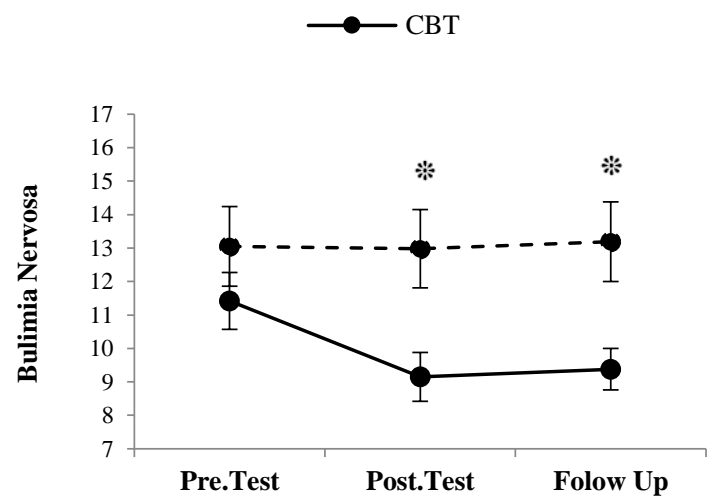

Figure 2. The Effect of Cognitive-behavioral Therapy on Signs of Bulimia Nervosa in Athletes with Bulimia Nervosa *: Significant Interactive Effect of Group and Time at $p<0.001$ Level

Valizade and Ariapooran examined the prevalence of eating disorders and their role in the psychological signs of women who exercise. The study results showed that $16.5 \%$ of athletes had symptoms of an eating disorder, $14.3 \%$ had signs of Anorexia Nervosa, and 3.3\% had signs of binge eating (34). Zeifodini examined eating disorders in athletic women and showed that $95 \%$ of eating disorders occur in women and $90 \%$ of them are under 25 years old. The prevalence of this disorder is between 0 to 27 percent in athletic women and 0 to 21 percent in the general population. $37 \%$ of athletes exercise for weight 
loss, $2.14 \%$ use a strict diet, and $10 \%$ induce vomiting and medication. The athletes who have low-calorie intake, such as vegetarians, and those who perform aesthetics sports such as ballet, scuba diving, and skiing, are more prone to these disorders. During these disorders and low energy intake, muscle strength and exercise performance are reduced, as well as morbid fatigue, and muscle cramps due to hypokalemia are observed (35).

In a study by Nobakht and Dezhkam, the prevalence of anorexia and binge-eating was $0.9 \%$ and $3.23 \%$, respectively, and marginal eating disorders were $6.6 \%$ (36). In a study by Mozaffari Khosravi, the prevalence of anorexia and binge-eating was 0.9 and $5.3 \%$ (37), respectively. In a survey by Seyedi and Sanaeizadeh, incidence of anorexia and bingeeating was 0.8 and 0.8 and eating mild disorder syndrome was $7 \%$ (38).

In explaining this result, it can be said that women who engage in exercise and physical activity are more likely to be concerned about their physical appearance, especially their weight and body, and this may lead to eating disorders such as anorexia and bulimia. In other words, worrying about losing weight may lead women to exercise such as aerobics and fitness, because various studies have also suggested a relationship between body weight and mental health concerns, and even between fear and anxiety. The women are more likely to have more eating disorders than others to lose weight and evaluate their bodybuilding criteria and are anxious about their physical future, and this anxiety encourages them to exercise as the easiest way to balance load. It can also be said that the high prevalence of eating disorders may also be related to cultural and social reasons because the emphasis on slimming, dieting and exercise is favored by all social classes and racial minorities and even families and is affected by occupational and nonoccupational and weight-related pressure (19).

Regarding the young population of Iran, the high prevalence of eating disorders is justified because, in general, the youth are more influenced by other cultures and care about the criteria of those cultures. In other words, in all cultures, weight and body fit is one of the requirements for beauty, and the social stress caused by weight loss has a more significant effect on the youth, and they like to adapt to beauty and weight loss criteria because proper bodybuilding can increase self-confidence and consequently improve interpersonal relationships and seek approval from others (34).

But the main result of the present study shows the effectiveness of cognitive-behavioral therapy on improving the signs of Bulimia Nervosa in the treatment group athletes compared to the control group. While the symptoms of Bulimia Nervosa in athletes with the disorder during cognitivebehavioral period were significantly reduced, there was no change in the athletes in the control group. On the other hand, after the end of the treatment period and during the follow-up period, the signs of Bulimia Nervosa in the two groups, as the post-test, remained unchanged. For the effectiveness of cognitive-behavioral therapy, we can refer to a study by Turner et al. In this study, it was shown that cognitive-behavioral therapy could modify the early signs of an eating disorder (1). The study results of Wilfley et al. also showed a significant reduction in symptoms of eating disorder through cognitive behavioral therapy, but the results were consistent with disease recurrence (39). In a study, Mahmoudiyandastnaee compared the effectiveness of schema therapy and cognitive behavioral therapy on eating disorder and stated that schema therapy has more lasting effects than cognitive behavioral therapy because cognitivebehavioral treatment is based on the patient's present time rather than the patient's past. Given that in the present study, the etiology of Bulimia Nervosa signs is related to the current situation, i.e. competitive conditions of the athlete, it seems appropriate to select cognitive behavioral therapy (40).

In this regard, Fairburn et al. in a prospective study examined the long-term effectiveness of three psychological treatments on some eating disorders and reported that half of the subjects had one of the eating disorders, a statistical and diagnostic guide to mental disorders, $19 \%$ were in Bulimia Nervosa class, 3\% had Anorexia Nervosa and $24 \%$ were classified as undiagnosed. There were few other psychiatric disorders, but importantly, those who received cognitive behavioral therapy were significantly better than those receiving behavioral treatment. The patients receiving behavioral treatment thus achieve shortterm outcomes, while the patients receiving cognitive behavioral therapy achieve long-term results (30).

In the present study, when athletes with Bulimia Nervosa under cognitive behavioral 
therapy experienced weight gain during the treatment period, the control group athletes with this disorder experienced weight loss. On the other hand, at the end of the treatment period and during the follow-up period, the two groups as the post-test remained unchanged. Turner et al., with the Cognitive Behavioral Therapy approach, examined the effectiveness of cognitivebehavioral therapy on outpatients with eating disorders. The results indicated a significant improvement in the pathology of the eating disorder, anxiety, depression, and total performance with marked changes in eating attitude at the early stages of treatment. The moderate effect size was shown. The results showed a significant improvement in subjects' eating attitudes and substantial changes in other topics (1). In a study, de Jong et al. investigated the effectiveness of cognitive-behavioral therapy for eating disorders, which is consistent with the present study (24).

Thompson and Sherman conducted extensive research on the origin and extent of eating disorders in athletes as well as possible solutions. The results showed that factors affecting the athletic environment that increase the risk of athlete's eating disorders include the belief of athletes and coaches in weight loss or body fat that can enhance athletic performance. The authors propose to change athletes' emphasis on sports performance for the athlete's health (13). This should be considered as one of the most important reasons for the changes observed in the present study because the cognitive-behavioral model for the treatment of eating disorders is based on the principle that dysfunctional beliefs about body weight and dissatisfaction with body shape and mass lead to and continue extravagant behaviors for nutrition and other prominent features such as vomiting and use of laxatives, diuretics and weight loss medications (21).

However, Mathisen et al. in a study examined the effectiveness of physical activity and diet therapy compared to cognitive behavioral therapy for treating patients with Bulimia Nervosa and Binge-eating and reported that the efficacy of diet therapy and physical activity in terms of behavioral and psychological signs was the same as cognitive behavioral therapy, but it was better in terms of reducing physical symptoms (5), as observed in the present study regarding weight changes. In this report, diet therapy and physical activity increased physical fitness, treatment process, and quality of life. As a result, diet therapy and physical activity showed good evidence as an alternative to cognitive behavioral therapy for the treatment of Bulimia Nervosa and Anorexia Nervosa (5), but the participants in the present study were athletes who had physical activity, so there is a need for behavior change through another approach. Turner pointed out that some athletes need to work at a deeper level to neutralize central irrational beliefs that lead to unhealthy behaviors and emotions that can be effectively addressed through rational emotional behavior therapy. Rational Emotional Behavior Therapy is humanistic and therefore not only focused on the athlete and his athletic performance, but also the person, therefore, cognitive-behavioral and emotional rational behavior therapy will be applicable for a wide range of muscular problems apart from functional issues such as career change, personal life problems and eating disorders (23).

\section{CONCLUSION}

For the first time, we investigated the effect of cognitive-behavioral therapy on athletes with signs of Bulimia Nervosa. Finally, after 16 weeks of cognitive-behavioral therapy (21 sessions), improvement of symptoms of Bulimia Nervosa and weight gain was observed in the athlete in the treatment group compared to the control group. At follow-up 12 weeks after treatment, these changes remained stable. Therefore, the results of the present study confirm the effectiveness of cognitivebehavioral therapy on the improvement of Bulimia Nervosa signs and weight management, as well as its persistence in athletes.

Although there is deficient awareness in the community of athletes, even among the general public, about eating disorders, they do not recognize it as a disorder or mental illness, so they do not follow for the treatment. As a result, coaches and athletes should pay more attention to the athletes' mental health so that appropriate measures and follow-up can be observed.

One of the limitations of the present study was the failure to record dietary intake of treated patients before, during, and after treatment to calculate the number of nutrients they received. Therefore, it is recommended to consider this in future research. 


\section{APPLICABLE REMARKS}

- The results of the present study confirm the effectiveness of Cognitive-Behavioral Therapy on the improvement of symptoms of athletes with Bulimia Nervosa; So:

- It is recommended that this protocol can be used for athletes with eating disorders, especially
Bulimia Nervosa, because of the effectiveness and stability of Cognitive-Behavioral Therapy.

- The Eating Disorder Diagnostic Scale (EDDS), along with a Semi-Structured Clinical Interview for DSM-IV Axis I Disorders (SCID-I), can be used in athletes to diagnose Bulimia Nervosa.

\section{REFERENCES}

1. Turner H, Marshall E, Wood F, Stopa L, Waller G. CBT for eating disorders: The impact of early changes in eating pathology on later changes in personality pathology, anxiety and depression. Behav Res Ther. 2016;77:1-6. doi: 10.1016/j.brat.2015.11.011 pmid: 26690743

2. Brown A, Mountford V, Waller G. Therapeutic alliance and weight gain during cognitive behavioural therapy for anorexia nervosa. Behav Res Ther. 2013;51(4-5):216-220. doi: 10.1016/j.brat.2013.01.008 pmid: 23435122

3. Dalle Grave R, Calugi S, Sartirana M, Fairburn CG. Transdiagnostic cognitive behaviour therapy for adolescents with an eating disorder who are not underweight. Behav Res Ther. 2015;73:79-82. doi: 10.1016/j.brat.2015.07.014 pmid: 26275760

4. APA. Diagnostic And Statistical Manual Of Mental Disorders. Fourth and Fifth ed. Washington, USA: American Psychiatric Association; 2013.

5. Mathisen TF, Rosenvinge JH, Pettersen G, Friborg O, Vrabel K, Bratland-Sanda S, et al. The PED$t$ trial protocol: The effect of physical exercise -and dietary therapy compared with cognitive behavior therapy in treatment of bulimia nervosa and binge eating disorder. BMC Psychiatry. 2017;17(1):180. doi: 10.1186/s12888-017-1312-4 pmid: 28494809

6. Mansrour S, Toozandehjani H. The Effectiveness of Dialectical Behavioral Therapy on Anxiety Sensitivity and Body Image in Female Bulimia Nervosa Patients. J Clin Psychol. 2019;11(1):37-48.

7. Brewer BW, Petrie TA. Psychopathology in sport and exercise. Washington, DC, US: American Psychological Association; 2002. 307-323 p.

8. Brownell KD, Rodin J, Wilmore JH. Eating, body weight, and performance in athletes: Lea \& Febiger; 1992.

9. Cogan K. Eating disorders: when rations become irrational. In: Murphy S, editor. The Sport Psychology Handbook: Human Kinetics; 2005. p. 237-254.

10. Dosil J, Díaz O. Valoración de la conducta alimentaria y de control del peso en practicantes de aeróbic. Rev Psicol Deporte. 2007;11(2).

11. Hulley AJ, Hill AJ. Eating disorders and health in elite women distance runners. Int J Eat Disord. 2001;30(3):312-317. doi: 10.1002/eat.1090 pmid: 11746292

12. Pruitt J, Kappius R, Imm P. Sports, Exercise, and Eating Disorders. In: Diamant L, editor. Psychology of Sports, Exercise, and Fitness: Social and Personal Issues. Washington: Hemisphere Publishing Corporation; 1991. p. 277.

13. Thompson RA, Trattner Sherman R. Athletes, Athletic Performance, and Eating Disorders: Healthier Alternatives. J Soc Issues. 2002;55(2):317-337. doi: 10.1111/0022-4537.00118

14. Warren BJ, Stanton AL, Blessing DL. Disordered eating patterns in competitive female athletes. Int J Eat Disord. 1990;9(5):565-569. doi: 10.1002/1098-108x(199009)9:5<565::aid-eat2260090512>3.0.co;2$\mathrm{n}$

15. Weinberg R, Gould D. Foundations of Sport and Exercise Psychology. 6th ed: Human Kinetics; 2014.

16. Kerr G, Berman E, Souza MJD. Disordered Eating in Women's Gymnastics: Perspectives of Athletes, Coaches, Parents, and Judges. J Appl Sport Psychol. 2006;18(1):28-43. doi: 10.1080/10413200500471301

17. Kirchner EM, Lewis RD, O'Connor PJ. Bone mineral density and dietary intake of female college gymnasts. Med Sci Sports Exerc. 1995;27(4):543-549. pmid: 7791585

18. Beck J, Beck A. Cognitive Behavior Therapy, Second Edition: Basics and Beyond: Guilford Publications; 2011. 
19. Sadock B, Sadock V, Ruiz P. Kaplan and Sadock's Synopsis of Psychiatry: Wolters Kluwer; 2014.

20. Field TA, Beeson ET, Jones LK. The New ABCs: A Practitioner's Guide to Neuroscience-Informed Cognitive-Behavior Therapy. J Ment Health Counsel. 2015;37(3):206-220. doi: 10.17744/1040-286137.3.206

21. Saekow J, Jones M, Gibbs E, Jacobi C, Fitzsimmons-Craft EE, Wilfley D, et al. StudentBodieseating disorders: A randomized controlled trial of a coached online intervention for subclinical eating disorders. Internet Interv. 2015;2(4):419-428. doi: 10.1016/j.invent.2015.10.004

22. Lutz W, Hofmann SG, Rubel J, Boswell JF, Shear MK, Gorman JM, et al. Patterns of early change and their relationship to outcome and early treatment termination in patients with panic disorder. $J$ Consult Clin Psychol. 2014;82(2):287-297. doi: 10.1037/a0035535 pmid: 24447004

23. Turner MJ. Rational Emotive Behavior Therapy (REBT), Irrational and Rational Beliefs, and the Mental Health of Athletes. Front Psychol. 2016;7:1423. doi: 10.3389/fpsyg.2016.01423 pmid: 27703441

24. de Jong M, Korrelboom K, van der Meer I, Deen M, Hoek HW, Spinhoven P. Effectiveness of enhanced cognitive behavioral therapy (CBT-E) for eating disorders: study protocol for a randomized controlled trial. Trials. 2016;17(1):573. doi: 10.1186/s13063-016-1716-3 pmid: 27914473

25. Dalle Grave R, Calugi S, Brambilla F, Abbate-Daga G, Fassino S, Marchesini G. The effect of inpatient cognitive-behavioral therapy for eating disorders on temperament and character. Behav Res Ther. 2007;45(6):1335-1344. doi: 10.1016/j.brat.2006.09.016 pmid: 17074299

26. Dosil J. Eating Disorders in Athletes. England: John Wiley \& Sons Ltd; 2008.

27. First MB, Gibbon M. The structured clinical interview for dsm-iv axis i disorders (scid-i) and the structured clinical interview for dsm-iv axis ii disorders (scid-ii). Comprehensive handbook of psychological assessment, Vol 2: Personality assessment. Hoboken, NJ, US2004. p. 134-143.

28. Amini H, Sharifi V, Asadi S, Mohammadi MR, Kaviani H, Semnani Y, et al. Validity of the Iranian version of the structured clinical interview for DSM-IV (SCID-I) in the diagnosis of psychiatric disorders. J Health Monitor. 2008;7(1):49-57.

29. Stice E, Bearman SK. Body-image and eating disturbances prospectively predict increases in depressive symptoms in adolescent girls: a growth curve analysis. Dev Psychol. 2001;37(5):597-607. doi: 10.1037//0012-1649.37.5.597 pmid: 11552756

30. Fairburn C. Cognitive Behavior Therapy and Eating Disorders. New York: The Guilford Press; 2008. 324 p.

31. Pernick Y, Nichols JF, Rauh MJ, Kern M, Ji M, Lawson MJ, et al. Disordered eating among a multiracial/ethnic sample of female high-school athletes. J Adolesc Health. 2006;38(6):689-695. doi: 10.1016/j.jadohealth.2005.07.003 pmid: 16730597

32. Petrie TA, Greenleaf C, Reel J, Carter J. Prevalence of eating disorders and disordered eating behaviors among male collegiate athletes. Psychol Men Mascul. 2008;9(4):267-277. doi: 10.1037/a0013178

33. Rosen LW, Hough DO. Pathogenic Weight-Control Behaviors of Female College Gymnasts. Phys Sportsmed. 1988;16(9):140-144. doi: 10.1080/00913847.1988.11709603 pmid: 27404154

34. Valizade A, Ariapooran S. Prevalence of Eating Disorders and Their Role in Psychological Signs among Women with Sport Activities. J Guilan Univ Med Sci. 2011;20(79):15-23.

35. Zeifodini A. Examining the Eating disorders among female athletes. Scientific Conference of Nutrition Students; Tehran, Iran: Iranian Food Support and Nutrition Association \& Islamic Azad University-Science and Research Branch; 2016.

36. Nobakht M, Dezhkam M. An epidemiological study of eating disorders in Iran. Int J Eat Disord. 2000;28(3):265-271. doi: 10.1002/1098-108x(200011)28:3<265::aid-eat3>3.0.co;2-1

37. Mozaffari Khosravi H, Hosseinzadeh Shamsi Anar M, Shariati Bafghi S, Mozaffari Khosravi V. Prevalence of Eating Disorders and Obesity in High School Girl Students in Yazd, 2010-2011. J Toloo-eBehdasht. 2011;10(1):38-49.

38. Seyedi F, Sanaeizadeh F. Prevalence of eating disorders among female high school students in Kerman. Kerman: Kerman University of Medical Sciences, 2002.

39. Wilfley DE, Welch RR, Stein RI, Spurrell EB, Cohen LR, Saelens BE, et al. A randomized comparison of group cognitive-behavioral therapy and group interpersonal psychotherapy for the treatment of overweight individuals with binge-eating disorder. Arch Gen Psychiatry. 2002;59(8):713-721. doi: 10.1001/archpsyc.59.8.713 pmid: 12150647 
40. Mahmoudiyandastnaee T, Amiri S, Neshatdoost H, Manshaei G. Comparing the effectiveness of schema therapy and CBT On modifying maladaptive schemas in patients16 to 23 years Bulimia nervosa considering the parental bonding. J Psychol Psychiatry. 2019;6(2):1-15. 